Publ. RIMS, Kyoto Univ.

15 (1979), 853-870

\title{
Finiteness Theorems on Weakly 1-complete Manifolds
}

\author{
By \\ Takeo OHSAWA*
}

Let $X$ be a complex manifold of (complex) dimension $n$ and $\pi: B \rightarrow$ $X$ be a holomorphic vector bundle over $X$. We consider the vector space of $C^{\infty} \bar{\partial}$ closed $B$-valued $(p, q)$ forms modulo $C^{\infty} \bar{\partial}$ exact $B$-valued forms, which we denote by $H^{p, q}(X, B)$. It is interesting and sometimes useful to know whether $H^{p, q}(X, B)$ is finite dimensional or not. Specifically, when $X$ is noncompact, the finite dimensionality of $H^{p, q}(X, B)$ is closely related to the function theoretic properties of $X$. The purpose of this article is to prove the following statement which was conjectured by S. Nakano:

If $X$ is reakly 1-complete and $B$ is positive outside a compact subset of $X$ and of rank $1, H^{n, q}(X, B)$ is finite dimensional for $q \geq 1$.

The author expresses his hearty thanks to Dr. A. Fujiki and Professor S. Nakano for their kind advices.

\section{$\S 1 . \quad$ Notations}

Let us fix the notations. We denote by $X$ a connected paracompact complex manifold of dimension $n$. We denote for a subset $K$ of $X$, the interior of $K$, the boundary of $K$ and the closure of $K$ by Int $K, \partial K$ and $\bar{K}$, respectively. For two subsets $K_{1}$ and $K_{2}$ of $X$, we mean by $K_{1} \Subset K_{2}$ that $\bar{K}_{1}$ is compact and contained in Int $K_{2}$. Let $\pi: B \rightarrow X$ be a holomorphic line bundle on $X$, and $\left\{U_{i}\right\}$ be an open covering of $X$ consisting of coordinate neighbourhoods $U_{i}$ with holomorphic coordinates $\left(z_{i}^{1}, \cdots, z_{i}^{n}\right)$, over which $\pi: B \rightarrow X$ is trivial, namely $\pi^{-1}\left(U_{i}\right)=U_{i} \times C$, and $\left(x, \zeta_{i}\right) \in U_{i} \times C$ and $\left(x, \zeta_{j}\right) \in U_{j} \times C$ represent the same point of $B$ if and only if

Received March 1, 1978.

* Research Institute for Mathematical Sciences, Kyoto University. 


$$
\zeta_{i}=e_{i j}(x) \zeta_{j}
$$

where $\left\{e_{i j}\right\}$ is a system of transition functions for $B$.

A B-valued differential form $f$ on $X$ is a system $\left\{f_{i}\right\}$ ofdiff erential forms defined on $U_{i}$, satisfying $f_{i}=e_{i j} f_{j}$ in $U_{i} \cap U_{j}$.

We denote by $C^{p, q}(X)\left(C^{p, q}(X, B)\right)$ the space of differential forms (resp. $B$-valued forms) of class $C^{\infty}$ and of type $(p, q)$ on $X$, and by $C_{0}^{p, q}(X)\left(C_{0}^{p, q}(X, B)\right)$ the space of the forms in $C^{p, q}(X)$ (resp. $\left.C^{p, q}(X, B)\right)$ with compact supports. For a subset $K$ of $X$, we denote by $C^{\infty}(\bar{K})$ the space of functions on $\bar{K}$ which are restrictions of $C^{\infty}$ functions defined on a neighbourhood of $\bar{K} . \quad C^{p, q}(\bar{K}, B)$ is defined similarly.

We fix a hermitian metric $d s^{2}$ in $X$, and a hermitian metric $\left\{a_{i}\right\}$ along the fibers of $B$. Here $a_{i}$ is a positive function such that

$$
a_{i}\left|e_{i j}\right|^{2}=a_{j} \text { in } U_{i} \cap U_{j} \text {. }
$$

For $f, g \in C^{p, q}(X, B)$, we set

$$
a_{i} f_{i} \wedge * \bar{g}_{i}=\langle f, g\rangle d v
$$

where $*$ is the star operator and $d v$ is the volume element with respect to the metric $d s^{2}$. $\langle f, g\rangle$ does not depend on $i$ and is a function defined on $X$. We have $\langle f, f\rangle \geq 0$. If either $f$ or $g \in C_{0}^{p, q}(X, B)$, then

$$
(f, g)_{\Psi}=\int_{X}\langle f, g\rangle e^{-t} d v
$$

is defined for any real valued function $\Psi$ of class $C^{\infty}$.

We have the operator

$$
\bar{\partial}: C^{p, q}(X, B) \rightarrow C^{p, q+1}(X, B)
$$

defined by $(\bar{\partial} f)_{i}=\bar{\partial} f_{i}$. We form the formal adjoint of $\bar{\partial}$ with respect to the inner product $(f, g)_{\Psi}$ in $C_{0}^{p, q}(X, B)$, and denote it by $\vartheta_{m}$.

We denote by $L^{p, q}(X, B, \Psi)$ the space of measurable $B$-valued forms $f$ of type $(p, q)$, square integrable in the sense that $(f, f)_{r}<\infty$. It is a Hilbert space with respect to the inner product $(f, g)_{4}$. We define

$$
\begin{aligned}
& \|f\|^{2}=(f, f)_{0} \\
& (f, g)=(f, g)_{0} \\
& \vartheta=\vartheta_{0}
\end{aligned}
$$




$$
L^{p, q}(X, B)=L^{p, q}(X, B, 0) .
$$

We also denote by $\bar{\partial}$ the smallest closed extension of

$$
\bar{\partial}: L^{p, q}(X, B, \Psi) \rightarrow L^{p, q+1}(X, B, \Psi) .
$$

In general, given two Hilbert space $H_{1}$ and $H_{2}$, and a closed linear operator $T: H_{1} \rightarrow H_{2}$ with dense domain, we denote its domain, range and nullity by $D_{T}, R_{T}$ and $N_{T}$, respectively. We denote the adjoint of $T$ by $T^{*}$. In the case when $H_{1}=L^{p, q-1}(X, B, \Psi), H_{2}=L^{p, q}(X, B, \Psi)$ and $T$ $=\bar{\partial}$, we let $D_{\bar{\partial}}=D_{\bar{\partial}}^{p, q-1}, R_{\bar{\partial}}=R_{\frac{p}{\bar{o}}, q}$ and $N_{\bar{\partial}}=N_{\bar{\partial}}^{p, q-1}$. We define $R_{\bar{\partial}}^{p, 0}$ to be $0 . \quad D_{\bar{\partial}^{*}}^{p, q}, R_{\bar{\partial}^{*}}^{p, q-1}$ and $N_{\bar{\partial}^{*}}^{p, q}$ are defined similarly.

\section{Definition 1. 1 .}

$$
{ }^{\prime} H^{p, q}(X, B, \Psi)=N_{\bar{\partial}}^{p, q} / \overline{R_{\bar{\partial}}^{p, q}},
$$

where we denote by $\overline{R_{\frac{\partial}{\partial}}^{p, q}}$ the closure of $R_{\frac{p}{\partial}}^{p, q}$ in $L^{p, q}(X, B, \Psi)$.

Note that ' $I H^{p, q}\left(X, B, \Psi^{r}\right)$ is a Hilbert space. We define

$$
\text { ' } H^{p, q}(X, B)={ }^{\prime} H^{p, q}(X, B, 0) .
$$

For a differential form $\xi$ on $X$, exterior multiplication of $\xi$ to $f$ $\in C^{p, q}(X, B)$ is defined by

$$
(e(\xi) \wedge f)_{i}=\xi \wedge f_{i} .
$$

Let $\omega$ be the fundamental form of the hermitian metric $d s^{2}$ on $X$. We define

$$
\begin{aligned}
& L=e(\omega) \\
& \Lambda=(-1)^{p_{+} q} * L * \quad \text { on } \quad C^{p, q}(X, B) .
\end{aligned}
$$

\section{§2. Weak Fimileness Theorem}

Definition 2. 1. $X$ is called weakly 1 -complete if there exists a $C^{\infty}$ plurisubharmonic function $\Psi$ on $X$ such that for any $c \in R$, where $R$ denotes the real numbers,

$$
X_{c}:=\{x ; \Psi(x)<c\} \supseteq X .
$$

We call such $\Psi$ an exhaustion function of $X$. Note that if $X$ is weakly 1 -complete $X$ has a countable base, so by Sard's theorem there is a nowhere dense subset $A \subset R$ such that if $c \in R-A, \partial\{x ; \Psi(x) \leq c\}$ 
is a smooth manifold of real dimension $2 n-1$.

Proposition 2.2. If $X$ is weakly 1-complete, then for any compact subset $K$ of $X$, there exists an exhaustion function $\Psi$ such that $\{x ; \Psi(x)=0\} \supset K$ and $\partial\{x ; \Psi(x)=0\}$ is smooth.

Proof) For any exhaustion function $\Phi$ there exists a $c \in R$ such that $\{x ; \Phi(x) \leq c\} \supseteq K$ and $\partial\{x ; \Phi(x) \leq c\}$ is smooth. We define $\lambda: R \rightarrow R$ as follows

$$
\begin{gathered}
\lambda(t)=0 \quad \text { if } t \leq c, \\
\lambda(t)=\exp \left(-\frac{1}{(t-c)^{2}}+t-c\right) \text { if } t \geq c .
\end{gathered}
$$

Then $\lambda(t)$ is a $C^{\infty}$ plurisubharmonic function vanishing in a neighbourhood of $\bar{K}$, and $\partial\{x ; \lambda(\Phi(x))=0\}$ is smooth, so we may take $\Psi:=\lambda(\Phi)$.

q.e.d.

Since $X$ is a paracompact manifold of class $C^{\infty}$ it has a hermitian metric $d s^{2}$. Let $\left\{a_{i}\right\}$ be a metric along the fibers of $B$.

Definition 2. 3. A holomorphic line bundle $\pi: B \rightarrow X$ is said to be positive on a subset $Y \subset X$, if there exists a coordinate cover $\left\{U_{i}\right\}$ of $X$ such that $\pi^{-1}\left(U_{i}\right)$ are trivial and the metric $\left\{a_{i}\right\}$ along the fibers of $B$ can be so chosen that

$$
\left(\frac{\partial^{2} \log \left(a_{i}^{-1}\right)}{\partial z_{i}^{\alpha} \partial \bar{z}_{i}^{\beta}}\right)>0 \quad \text { on } \quad U_{i} \cap Y \quad \text { for any } i
$$

From now on, we let $X$ be a weakly 1-complete manifold and $\pi: B \rightarrow X$ be a holomorphic line bundle which is positive on the complement of a compact subset $K$ of $X$, and an exhaustion function $\Psi$ is so fixed that $K \Subset\{x ; \Psi(x)=0\}$ and $\partial\{x ; \Psi(x)=0\}$ is smooth. For convenience we denote $\operatorname{Int}\{x ; \Psi(x)=0\}$ by $X_{0}$.

Lemma 2. 4. For any finite number of $B$-valued forms $f^{1}, \cdots$, $f^{m}$ which are measurable and locally square integrable there exist a hermitian metric $d s^{2}$ and a metric $\left\{a_{i}\right\}$ along the fibers of $B$ such that 
1) $d s^{2}$ is a complete hermitian metric

2) $d s^{2}=\sum_{\alpha, \beta} \frac{\partial^{2} \log \left(a_{i}^{-1}\right)}{\partial z_{i}^{\alpha} \partial \bar{z}_{i}^{\beta}}\left(d z_{i}^{a}, d \bar{z}_{i}^{\beta}\right) \quad$ on $\quad X-K_{1}$

3) $\left(f^{l}, f^{l}\right)<\infty$, for $1 \leq l \leq m$,

for some compact set $K_{1}$ with $K \Subset K_{1} \Subset X_{0}$.

Proof) By the hypothesis on $B$ there exist $d s_{0}^{2}$ and $\left\{a_{i}^{0}\right\}$ which satisfy 2) for some $K_{1}$ with $K \Subset K_{1} \Subset X_{0}$. We define $d s^{2}$ and $\left\{a_{i}\right\}$ by

$$
\begin{gathered}
d s^{2}=\sum_{\alpha, \beta} \frac{\partial^{2} \log \left(a_{i}^{-1}\right)}{\partial z_{i}^{\alpha} \partial \bar{z}_{i}^{\beta}}\left(d z_{i}^{\alpha}, d \bar{z}_{i}^{\beta}\right) \text { on } X-K_{1}, \\
d s^{2}=d s_{0}^{2} \quad \text { on } K_{1} \\
a_{i}=a_{i}^{0} e^{-\tau(\Psi)},
\end{gathered}
$$

where $\tau$ is a real valued function of class $C^{\infty}$ such that

$$
\begin{aligned}
& \tau(t) \geq 0, \\
& \tau^{\prime}(t) \geq 0, \quad \text { and } \\
& \tau^{\prime \prime}(t) \geq 0 \quad \text { for any } t \in R .
\end{aligned}
$$

We set

$$
\begin{aligned}
& g_{i, \alpha \beta}=\frac{\partial^{2} \log \left(\left(a_{i}^{0}\right)^{-1}\right)}{\partial z_{i}^{\alpha} \partial \bar{z}_{i}^{\beta}} \\
& \Gamma_{i, \alpha \beta}=\frac{\partial^{2} \log \left(a_{i}^{-1}\right)}{\partial z_{i}^{\alpha} \partial \bar{z}_{i}^{\beta}} \\
& a_{i}^{0} f_{i} \wedge * \bar{f}_{i}=a^{0}(f) d v_{0} \\
& a_{i} f_{i} \wedge \text { } \bar{f}_{i}=a(f) d v=A(f) d v_{0},
\end{aligned}
$$

where $*$, $t$, and $d v_{0}, d v$ are the star operators and the volume forms of $d s_{0}^{2}$ and $d s^{2}$, respectively. We have by a direct calculation

$$
\begin{gathered}
A(f) \leq e^{-\tau(\Psi)} \frac{\operatorname{det}\left(\Gamma_{i, \alpha \beta}\right)}{\operatorname{det}\left(g_{i, \alpha \beta}\right)} a^{0}(f) \quad \text { on } \quad X-K_{1} \\
\frac{\operatorname{det}\left(\Gamma_{i, \alpha \beta}\right)}{\operatorname{det}\left(g_{i, \alpha \beta}\right)} \leq\left(1+\tau^{\prime}(\Psi)\right) v^{n}+\left(1+\tau^{\prime}(\Psi) v\right)^{n-1} \tau^{\prime \prime}(\Psi) u
\end{gathered}
$$

where $v$ and $u$ are non-negative continuous functions independent of $\tau$. We choose a non-decreasing continuous function $\rho(t)$ such that 


$$
\int_{X} e^{-\rho(\Psi)} a^{0}\left(f^{l}\right) d v_{0}<\infty, \quad \text { for } \quad 1 \leq l \leq m
$$

We use the following lemma due to S. Nakano.

Sublemma 2.5 ([6], Lemma). Given a real valued, continuous and strictly increasing function $\lambda(t)$ on $0 \leq t<\infty$ rvith $\lambda(0)=0$, $\lambda(t) \rightarrow \infty$ for $t \rightarrow \infty$, we can find a $C^{\infty}$ function $\tau(t)$ on $-\infty<t<\infty$ such that

$$
\begin{aligned}
& \tau^{\prime}(t) \geq 0 \quad \text { and } \quad \tau^{\prime \prime}(t) \geq 0, \quad \text { for any } t, \\
& \tau(t) \geq \lambda(t) \quad \text { for } t \geq c, \quad \text { and } \\
& \tau^{\prime}(t) \leq K \tau(t)^{2} \quad \text { and } \\
& \tau^{\prime \prime}(t) \leq K \tau(t)^{3} \quad \text { for } t \geq c^{\prime},
\end{aligned}
$$

for some constants $c, c^{\prime}$ and $K>0$.

We apply it to

$$
\lambda(t)=\max \{v(t), u(t)\}+2 \rho(t)+t^{2}
$$

and take $\tau(t)$ as in Lemma 2.5. Since

$$
\int_{0}^{\infty} \sqrt{\tau^{\prime \prime}(t)} d t=\infty
$$

$d s^{2}$ is complete ([5], Proposition 1). By (30) it follows that

$$
\begin{aligned}
& \int_{X-K_{1}} \exp (-\tau(\Psi)) \frac{\operatorname{det}\left(\Gamma_{i, \alpha \beta}\right)}{\operatorname{det}\left(g_{i, \alpha \beta}\right)} a^{0}(f) d v_{0} \\
& \leq \sup _{X-K_{1}} \exp \left(-\frac{\tau(\Psi)}{2}\right) \frac{\operatorname{det}\left(\Gamma_{i, \alpha \beta}\right)}{\operatorname{det}\left(g_{i, \alpha \beta}\right)} \\
& \quad \times \int_{X-K_{1}} \exp (-\rho(\Psi)) a^{0}(f) d v_{0}, \text { for } 1 \leq l \leq m .
\end{aligned}
$$

It is clear that for such $\tau$ the metrics $d s^{2}$ and $\left\{a_{i}\right\}$ satisfy 1), 2) and 3).

Theorem 2.6. Assume that $d s^{2}$ and $\left\{a_{i}\right\}$ satisfy 1) and 2) of Lemma 2. 4, then if $p+q>n$ 


$$
R^{p, q} \text { is closed }
$$

and

$$
\operatorname{dim}_{C}{ }^{\prime} H^{p, q}(X, B)<\infty .
$$

Proof) We use the following lemma which is theorem 1.1.3 of $[2]$.

Lemma 2.7. Let $H_{i}(i=1,2,3)$ be three Hilberl spaces and $T: H_{1} \rightarrow H_{2}$ and $S: H_{2} \rightarrow H_{3}$ be closed linear operators with dense domains such that $S T=0$. Assume that for any sequence $\left\{f_{\nu}\right\}$ with $f_{\nu} \in H_{2} \cap D_{S} \cap D_{T},\left\|f_{\nu}\right\|_{H_{2}} \leq 1, \lim _{\nu \rightarrow \infty}\left\|S f_{\nu}\right\|_{H_{3}}=0$, and $\lim _{\nu \rightarrow \infty}\left\|T f_{\nu}\right\|_{H_{1}}=0$, we can choose a strongly convergent subsequence of $\left\{f_{\nu}\right\}$. Then $R_{T}$ is closed and $N_{S} / R_{T}$ is a finite dimensional vector space.

According to Lemma 2.7, in order to prove (38) and (39) it suffices to show that for any sequence $\left\{f_{\nu}\right\}$ such that $f_{\nu} \in D_{\overline{\bar{\partial}}}^{p, q} \cap D_{\bar{\partial}^{*}}^{p, q}$, $\left\|f_{\nu}\right\| \leq 1, \lim _{\nu \rightarrow \infty}\left\|\bar{\partial} f_{\nu}\right\|=0$, and $\lim _{\nu \rightarrow \infty}\left\|\bar{\partial} * f_{\nu}\right\|=0$, we can choose a strongly convergent subsequence of $\left\{f_{\nu}\right\}$.

Since $d s^{2}$ is complete, $C_{0}^{p, q}(X, B)$ is a dense subset of $D_{\frac{p}{\partial}}^{p, q} \cap D_{\bar{\partial}^{*}}^{p, q}$ with respect to the graph norm,

$$
\left\{(\bar{\partial} f, \bar{\partial} f)+\left(\bar{\partial}^{*} f, \bar{\partial}^{*} f\right)+(f, f)\right\}^{1 / 2}
$$

([8], Theorem 1.1). Hence we may assume

$$
f_{\nu} \in C_{0}^{p, q}(X, B) \text {. }
$$

Therefore, we have

$$
\begin{gathered}
\left(\bar{\partial} f_{\nu}, \bar{\partial} f_{\nu}\right)+\left(\bar{\partial}^{*} f_{\nu}, \bar{\partial}^{*} f_{\nu}\right)+\left(f_{\nu}, f_{\nu}\right) \\
=\left((\bar{\partial} \vartheta+\vartheta \bar{\partial}) f_{\nu}, f_{\nu}\right)+\left(f_{\nu}, f_{\nu}\right) .
\end{gathered}
$$

Hence by the assumption

$$
\left((\bar{\partial} \vartheta+\vartheta \bar{\partial}) f_{\nu}, f_{\nu}\right)+\left(f_{\nu}, f_{\nu}\right)
$$

is bounded above, which combined with ellipticity of $\bar{\partial} \vartheta+\vartheta \bar{\partial}$ means that $\left(f_{y}\right)_{i}$ and their first order derivatives are bounded. Here the derivatives are taken with respect to the coordinate of $U_{i}$. Combining this with 
Rellich's lemma, it follows that $\left\{f_{\nu}\right\}$ has a subsequence $\left\{f_{\nu_{j}}\right\}$ which is strongly convergent on compact subsets. We use the following estimate which is proved later (See Lemma 3.3 in Section 3.)

$$
\int_{X-K_{2}}\langle f, f\rangle d v \leq C\left\{\|\bar{\partial} f\|^{2}+\|\vartheta f\|^{2}+\int_{K_{2}}\langle f, f\rangle d v\right\},
$$

if $p+q>n$ and $f \in D_{\bar{\partial}}^{p, q} \cap D_{\vartheta}^{p, q}$, where $C$ is a constant and $K_{1} \Subset K_{2} \Subset X_{0}$. By this estimate we conclude that $\left\{f_{\nu,}\right\}$ converges strongly on $X$.

q.e.d.

\section{$\S$ 3. The Basic Estimate}

In what follows $d s^{2}$ and $\left\{a_{i}\right\}$ are assumed to satisfy 1) and 2) of Lemma 2. 4.

For an integer $\nu$, we define

$$
\begin{aligned}
& (f, g)_{\nu}=(f, g)_{\nu \Psi}, \quad \text { and } \\
& \|f\|_{\nu}^{2}=(f, f)_{\nu}, \quad \text { for } f, g \in L^{p, q}(X, B, \nu \Psi) .
\end{aligned}
$$

We let $\vartheta$, be the formal adjoint of $\bar{\partial}$ with respect to the inner product $(f, g)$, and we define

$$
\square_{\nu}=\bar{\partial} \vartheta_{\nu}+\vartheta_{\nu} \bar{\partial}
$$

Since $d s^{2}$ is complete $\vartheta_{\nu}$ is equal to the adjoint of $\bar{\partial}$. ([8], Theorem 1. 1).

We use a well known formula in differential geometry in the following form.

\section{Lemma 3. 1.}

$$
\square_{\nu}-*^{-1} \square_{\nu} *=e\left(\chi_{\nu}\right) \Lambda-\Lambda e\left(\chi_{\nu}\right), \quad \text { on } \quad X-K_{1}
$$

where $e\left(\chi_{\nu}\right)=L+e(\nu \sqrt{-1} \partial \bar{\partial} \Psi)$.

Proof) We let

$$
\begin{aligned}
& b_{i}=e^{-\nu T} a_{i}, \\
& D=d+\partial \log b_{i}, \quad \text { and } \\
& D^{\prime}=\partial+\partial \log b_{i} .
\end{aligned}
$$

Then we have 


$$
\begin{gathered}
e\left(\chi_{\nu}\right)=\sqrt{-1} D^{2}, \quad \text { and } \\
D^{2}=\left(\bar{\partial}+D^{\prime}\right)\left(\bar{\partial}+D^{\prime}\right)=\bar{\partial} D^{\prime}+D^{\prime} \bar{\partial} .
\end{gathered}
$$

Letting $\delta^{\prime}$ be the formal adjoint of $\bar{\partial}: C_{0}^{p, q}(X) \rightarrow C_{0}^{p, q+1}(X)$ we have

$$
\sqrt{ }-1(\bar{\partial} \Lambda-\Lambda \bar{\partial})=\delta^{\prime} \quad \text { on } \quad X-K_{1} \text {. }
$$

We have

$$
\sqrt{ }-1\left(D^{\prime} \Lambda-\Lambda D^{\prime}\right)=\vartheta_{\nu} \quad \text { on } \quad X-K_{1} \text {. }
$$

Hence

$$
\begin{aligned}
e\left(\chi_{\nu}\right) \Lambda & -\Lambda e\left(\chi_{\nu}\right) \\
= & \sqrt{ }-1\left\{\left(\bar{\partial} D^{\prime}+D^{\prime} \bar{\partial}\right) \Lambda-\Lambda\left(\bar{\partial} D^{\prime}+D^{\prime} \bar{\partial}\right)\right\} \\
= & \sqrt{ }-1\left\{\left(\bar{\partial}\left(D^{\prime} \Lambda-\Lambda D^{\prime}\right)+\left(D^{\prime} \Lambda-\Lambda D^{\prime}\right) \bar{\partial}\right.\right. \\
& \left.-D^{\prime}(\bar{\partial} \Lambda-\Lambda \bar{\partial})-(\bar{\partial} \Lambda-\Lambda \bar{\partial}) D^{\prime}\right\} \\
= & \bar{\partial} \vartheta_{\nu}+\vartheta_{\nu} \bar{\partial}-\left(D^{\prime} \delta^{\prime}+\delta^{\prime} D^{\prime}\right) \\
= & \square_{\nu}-*^{-1} \square_{\nu} * \text { on } X-K_{1},
\end{aligned}
$$

\section{Lemma 3. 2.}

$$
\|f\|^{2} \leq\|\bar{\partial} f\|^{2}+\|\vartheta f\|^{2}
$$

if $f \in C_{0}^{p, q}\left(X-K_{1}, B\right)$ and $p+q>n$, and

$$
\|f\|_{\nu}^{2} \leq\|\bar{\partial} f\|_{\nu}^{2}+\left\|\vartheta_{\nu} f\right\|_{\nu}^{2}
$$

if $f \in C_{0}^{n, q}\left(X-K_{1}, B\right)$ and $q \geq 1$.

Proof) We prove (58) first. If $f \in C_{0}^{n, q}\left(X-K_{1}, B\right)$ and $q \geq 1$, we have

$$
\begin{aligned}
\|\bar{\partial} f\|_{\nu}^{2}+ & \left\|\vartheta_{\nu} f\right\|_{\nu}^{2}=\left(\square_{\nu} f, f\right)_{\nu} \\
\geq & \left(\left(e\left(\chi_{\nu}\right) \Lambda-\Lambda e\left(\chi_{\nu}\right)\right) f, f\right)_{\nu} \\
= & ((L \Lambda-\Lambda L) f, f)_{\nu} \\
& +\nu(e(\sqrt{ }-1 \partial \bar{\partial} \Psi) \Lambda-\Lambda e(\sqrt{ }-1 \partial \bar{\partial} \Psi)) f, f)_{\nu} \\
= & q(f, f)_{\nu}+\nu(e(\sqrt{ }-1 \partial \bar{\partial} \Psi) \Lambda f, f)_{\nu} \geq q(f, f)_{\nu} .
\end{aligned}
$$

For more details, see [5]. However we note that what is proved there is 
that if the dual of $B$ is positive and $\varphi$ is a $(0, n-q)$ form with support contained in a coordinate neighbourhood $U$, we have

$$
((\Lambda e(\sqrt{-1} \partial \bar{\partial} \Psi)-e(\sqrt{-1} \partial \bar{\partial} \Psi) \Lambda) \varphi, \varphi) \geq 0
$$

so it seems not sufficient to establish (60) for the elements of $C_{0}^{0, n-q}(X, B)$. But his argument also implies

$$
\langle\Lambda e(\sqrt{-1} \bar{\partial} \bar{\partial} \Psi) \varphi, \varphi\rangle \geq 0
$$

for $\varphi \in C^{0, n-q}(X, B)$. Hence rewriting (61) we obtain (58).

The proof of (57) is similar as above. q.e.d.

Lemma 3. 3. there is a constant $C$ and a compact set $K_{2}$ with $K_{1} \Subset K_{2} \Subset X_{0}$ such that

$$
\int_{X-K_{2}}\langle f, f\rangle e^{-\nu \Psi} d v \leq C\left\{\|\bar{\partial} f\|_{\nu}^{2}+\left\|\vartheta_{\nu} f\right\|_{\nu}^{2}+\int_{K_{2}}\langle f, f\rangle d v\right\}
$$

if $\nu \geq 0, f \in D_{\bar{\partial}}^{n, q} \cap D_{\vartheta_{\nu}}^{n, q}$ and $q \geq 1$, and

$$
\int_{X-K_{2}}\langle f, f\rangle d v \leq C\left\{\|\bar{\partial} f\|^{2}+\|\vartheta f\|^{2}+\int_{K_{2}}\langle f, f\rangle d v\right\}
$$

if $f \in D_{\frac{p}{\partial}, q} \cap D_{\vartheta}^{p, q}$ and $p+q>n$.

Proof) Since $d s^{2}$ is complete we may assume $f \in C_{0}^{p, q}(X, B)$. We prove (63). The proof of (62) is similar.

Let $\chi$ be a $C^{\infty}$ function on $Y$ such that for a compact set $K_{2}$ with $K_{1} \Subset K_{2} \Subset X_{0}$

$$
\begin{aligned}
& \chi=1 \quad \text { in } X-K_{2}, \text { and } \\
& \chi=0 \quad \text { in a neighbourhood of } K_{1} .
\end{aligned}
$$

We have $\chi f \in C_{0}^{n, q}\left(X-K_{1}, B\right)$, so we can apply Lemma 3. 2, getting

$$
\|\chi f\|_{\nu}^{2} \leq\|\bar{\partial}(\chi f)\|_{\nu}^{2}+\left\|\vartheta_{\nu}(\chi f)\right\|_{\nu}^{2} .
$$

We estimate the both sides of this inequality. The left hand side:

$$
\int_{X-K_{2}}\langle f, f\rangle e^{-\nu T} d v \leq\|\chi f\|_{\nu}^{2} .
$$

The right hand side:

$$
\begin{aligned}
& \|\bar{\partial}(\chi f)\|_{\nu}^{2}+\left\|\vartheta_{\nu}(\chi f)\right\|_{\nu}^{2} \\
= & \|\bar{\partial} \chi \Lambda f+\chi \bar{\partial} f\|_{\nu}^{2}+\left\|-* a_{i}^{-1} e^{\nu \psi} \bar{\partial} * a_{i} e^{-\nu T}(\chi f)\right\|_{\nu}^{2}
\end{aligned}
$$


Finiteness Theorems on WeAKLy 1-COMPLete Manifolds

$$
\begin{aligned}
=\| & \bar{\partial} \chi \Lambda f+\chi \bar{\partial} f\left\|_{\nu}^{2}+\right\| \chi \vartheta_{\nu} f-*(\partial \chi \Lambda * f) \|_{\nu}^{2} \\
\leq & \|\bar{\partial} \chi \Lambda f\|_{\nu}^{2}+2 \operatorname{Re}(\bar{\partial} \chi \Lambda f, \chi \bar{\partial} f)_{\nu}+\|\chi \bar{\partial} f\|_{\nu}^{2}+\left\|\chi \vartheta_{\nu} f\right\|_{\nu}^{2} \\
& -2 \operatorname{Re}\left(\chi \vartheta_{\nu} f, *(\partial \chi \Lambda * f)\right)_{\nu}+\|*(\partial \chi \Lambda * f)\|_{\nu}^{2} \\
\leq & \int_{K_{2}}\langle\bar{\partial} \chi, \bar{\partial} \chi\rangle d v \cdot \int_{K_{2}}\langle f, f\rangle d v+\|\bar{\partial} \chi \Lambda f\|_{\nu}^{2}+\|\chi \bar{\partial} f\|_{\nu}^{2} \\
& +\|\chi \bar{\partial} f\|_{\nu}^{2}+\left\|\chi \vartheta_{\nu} f\right\|_{\nu}^{2}+\left\|\chi \vartheta_{\nu} f\right\|_{\nu}^{2}+\|*(\partial \chi \Lambda * f)\|_{\nu}^{2} \\
& +\int_{K_{2}}\langle\bar{\partial} \chi, \bar{\partial} \chi\rangle d v \cdot \int_{K_{2}}\langle f, f\rangle d v \\
\leq & 2\left(\int_{K_{2}}\{\langle\bar{\partial} \chi, \bar{\partial} \chi\rangle+\langle\partial \chi, \partial \chi\rangle\} d v \cdot \int_{K_{2}}\langle f, f\rangle d v\right) \\
& +2 \sup _{x \in X} \chi(x) \cdot\left(\|\bar{\partial} f\|_{\nu}^{2}+\left\|\vartheta_{\nu} f\right\|_{\nu}^{2}\right) .
\end{aligned}
$$

Therefore, if

$$
C \geq 2 \max \left(\int_{K_{2}}\{\langle\bar{\partial} \chi, \bar{\partial} \chi\rangle+\langle\partial \chi, \partial \chi\rangle\} d v, \sup _{x \in X} \chi(x)\right)
$$

we have

$$
\int_{X-K_{2}}\langle f, f\rangle e^{-\nu T} d v \leq C\left(\|\bar{\partial} f\|_{\nu}^{2}+\left\|\vartheta_{\nu} f\right\|_{\nu}^{2}+\int_{K_{2}}\langle f, f\rangle d v\right)
$$

q.e.d.

\section{\$4. The Main Theorem}

Defimition 4. We denote by $\mathcal{H}^{p, q}\left(X_{0}, B\right)$ the set of elements $h \in L^{p, q}\left(X_{0}, B\right)$ with $\bar{\partial} h=0$ and $\bar{\partial}^{*} h=0$, where $\bar{\partial}^{*}$ is the adjoint of $\bar{\partial}: L^{p, q-1}\left(X_{0}, B\right) \rightarrow L^{p, q}\left(X_{0}, B\right)$.

Proposition 4.2. There exist $\nu_{0}$ and $C_{0}$ such that for any $\nu \geq \nu_{0}$

$$
\|f\|_{\nu}^{2} \leq C_{0}\left(\|\bar{\partial} f\|_{\nu}^{2}+\left\|\vartheta_{\nu} f\right\|_{\nu}^{2}\right), \text { where }
$$

provided

$$
f \in L^{n, q}(X, B, \nu \Psi) \cap D_{\vec{\partial}}^{n, q} \cap D_{\mho_{\nu}}^{n, q}(q \geq 1)
$$

and

$$
\int_{X_{0}}\langle f, h\rangle d v=0 \text { for any } h \in \mathcal{H}^{n, q}\left(X_{0}, B\right)
$$


Proof) If the proposition were false, we may assume that there is a sequence $\left\{f_{\nu}\right\}$ such that

$$
\left\|f_{\nu}\right\|_{\nu}^{2}=1
$$

$$
\left\|\bar{\partial} f_{\nu}\right\|_{\nu}^{2}+\left\|\vartheta_{\nu} f_{\nu}\right\|_{\nu}^{2} \leq \frac{1}{\nu}
$$

$$
f_{\nu} \in L^{n, q}(X, B, \nu \Psi) \cap D_{\vec{\partial}}^{n, q} \cap D_{\vartheta_{\nu}}^{n, q}
$$

and

$$
\int_{X_{0}}\left\langle f_{\nu}, h\right\rangle d v=0 \quad \text { for any } \quad h \in \mathcal{I}^{n, q}\left(X_{0}, B\right) .
$$

Let $g_{\nu}=e^{-\nu T} f_{\nu}$, then we have

$$
\vartheta g_{\nu}=e^{-\nu T} f_{\nu}
$$

so that

$$
\left\|\vartheta g_{\nu}\right\|_{-\nu}=\left\|\vartheta_{\nu} f_{\nu}\right\|_{\nu}
$$

hence

$$
\lim _{\nu \rightarrow \infty}\left\|\vartheta g_{\nu}\right\|_{-\nu}=\lim _{\nu \rightarrow \infty}\left\|\vartheta_{\nu} f_{\nu}\right\|_{\nu}=0
$$

by (75). Since $\left\|\vartheta g_{\nu}\right\| \leq\left\|\vartheta g_{\nu}\right\|_{-\nu}$, we have $\lim _{\nu \rightarrow \infty}\left\|\vartheta g_{\nu}\right\|=0$. By (74), we have

$$
\left\|g_{\nu}\right\|_{-\nu}=\left\|f_{\nu}\right\|_{\nu}=1
$$

hence $\left\|g_{\nu}\right\| \leq 1$. Therefore choosing a subsequence, we may assume that $\left\{g_{\nu}\right\}$ has a weak limit $g$ in $L^{n, q}(X, B)$. It is easily verified that

$$
\|g\|_{-\nu} \leq \inf _{\mu \geq \nu} \sup _{\mu^{\prime} \geq \nu}\left\|g_{\mu^{\prime}}\right\|_{-\mu^{\prime}}=1
$$

for any $\nu \geq 1$. Thus we have supp $g \Subset X_{0}$. Therefore,

$$
\bar{\partial} *\left(\left.g\right|_{X_{0}}\right)=0 \text {. }
$$

(See Appendix.) By (75) $\bar{\partial} g=0$, and $g$ satisfies (77). By (74) and (75), it may be assumed that $\left\{g_{\nu}\right\}$ is strongly convergent on $K_{2}$, and the limit is not zero on $K_{2}$ by Lemma 3.3. Hence we conclude that $\left.g\right|_{X_{0}} \neq 0$. This contradiction completes the proof. q.e.d. 


\section{Definition 4.3.}

$$
H_{l o c}^{p, q}(X, B)=\frac{\left\{f ; f \in L_{l o c}^{p, q}(X, B), \bar{\partial} f=0\right\}}{-\overline{L_{l o c}^{p, q}}(X, B) \cap\left\{\bar{\partial} g ; g \in L^{\bar{p}, q-1}(X, B)\right\}}
$$

where we denote by $L_{l o c}^{p, q}(X, B)$ the space of $B$-valued $(p, q)$ forms which are measurable and square integrable on compact subsets of $X$.

By the Dolbeault's theorem (see [2], Theorem 2.2.4 and Theorem 2. 2. 5), there is a natural isomorphism between the spaces $H_{1 \mathrm{loc}}^{p, q}(X, B)$ and $H^{p, q}(X, B)$.

We define ' $H^{p, q}\left(X_{0}, B\right)$ with respect to $d s^{2}$ and $\left\{a_{i}\right\}$.

Proposition 4. 4. The natural map

$$
\rho: H_{l o c}^{n, q}(X, B) \rightarrow^{\prime} H^{n, q}\left(X_{0}, B\right)
$$

is injective for $q \geq 1$.

Proof) We show that if $f \in L_{l o c}^{n, q}(X, B), \bar{\partial} f=0$, and there exists a sequence $\left\{g_{\nu}\right\}$ with $g_{\nu} \in L^{n, q-1}\left(X_{0}, B\right), \bar{\partial} g_{\nu} \in L^{n, q}\left(X_{0}, B\right)$ and

$$
\int_{X_{0}}\left\langle f-\bar{\partial} g_{\nu}, f-\bar{\partial} g_{\nu}\right\rangle d v<\frac{1}{\nu}
$$

then there exists $g \in L_{l o c}^{n, q-1}(X, B)$ such that $\bar{\partial} g=f$.

We replace the exhaustion function $\Psi$ by $\widetilde{\Psi}=\lambda(\Psi)$ where $\lambda$ is a convex increasing $C^{\infty}$ function with $\lambda(0)=0$ and $\lambda(t)>0$ if $t>0$ which increases so rapidly that

$$
\int_{X}\langle f, f\rangle e^{-\widetilde{\Psi}} d v<\infty .
$$

For $\nu \geq 1$, we have $f \in L^{n, q}(X, B, \nu \widetilde{\Psi})$.

By (86) it follows that

$$
\int_{X_{0}}\langle f, h\rangle d v=0 \quad \text { for any } h \in \mathcal{M}^{n, q}\left(X_{0}, B\right) .
$$

Therefore, combining Proposition 4.2 with Hörmander's theorem ([2], Theorem 1.1.4) we conclude that for some $\nu \geq 1$ there exists $g \in$ $L^{n, q-1}(X, B, \nu \widetilde{\Psi})$ such that $\bar{\partial} g=f$.

q.e.d.

We fix $c>0 . \quad X_{c}$ is a weakly 1-complete manifold with an exhaustion 
function

$$
\Psi_{c}=\frac{1}{c-\Psi}
$$

We choose a hermitian metric $d \sigma^{2}$ and a metric $\left\{b_{i}\right\}$ along the fibers of $B$ such that

i) $\quad d \sigma^{2}$ is complete

ii) $\quad d \sigma^{2}=\sum_{\alpha, \beta} \frac{\partial^{2} \log \left(b_{i}^{-1}\right)}{\partial z_{i}^{\alpha} \partial \bar{z}_{i}^{\beta}}\left(d z_{i}^{\alpha}, d \bar{z}_{i}^{\beta}\right) \quad$ on $\quad X-K_{1}$.

Moreover we may assume that

iii)

$$
\int_{X_{0}} b_{i} f_{i} \wedge \nLeftarrow \bar{f}_{i}<\infty, \quad \text { for } \quad f \in C^{p, q}(X, B),
$$

where is the star operator for $d \sigma^{2}$. This follows from Lemma 2.4 and that $C^{p, q}\left(\bar{X}_{c}, B\right)$ is a finitely generated $C^{\infty}\left(\bar{X}_{c}\right)$ module. We define ' $H^{p, q}\left(X_{c}, B\right)$ with respect to $d \sigma^{2}$ and $\left\{b_{i}\right\}$.

Theorem 4.5.

$$
\operatorname{dim}_{C} H^{n, q}(X, B)<\infty \quad \text { for } \quad q \geq 1 .
$$

Proof) Since ' $H^{n, q}\left(X_{c}, B\right)$ is finite dimensional it suffices to show that the natural map (induced by the restriction of forms)

$$
r: H^{n, q}(X, B) \rightarrow^{\prime} H^{n, q}\left(X_{c}, B\right)
$$

is injective. (Note that $r$ is well defined by the choice of $d \sigma^{2}$ and $\left\{b_{i}\right\}$. We consider the following diagram.

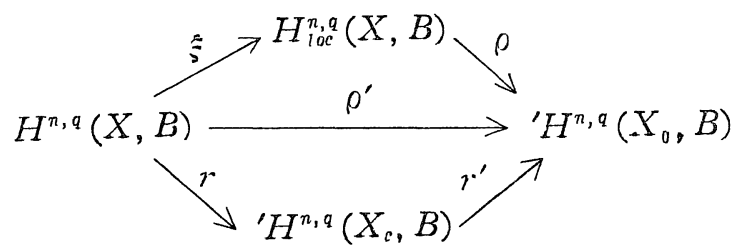

where $\rho^{\prime}, r^{\prime}$ and $\xi$ are natural homomorphisms. Since $\rho$ is injective and $\xi$ is an isomorphism, $\rho^{\prime}$ is injective, hence $r$ is injective. q.e.d, 
Theorem 4. 6. If $g \in L^{n, q}\left(X_{0}, B\right) \quad(q \geq 0)$ and $\bar{\partial} g=0$, then for any $\varepsilon>0$ there exists $f \in L_{l o c}^{n, q}(X, B)$ such that

$$
\int_{X_{0}}\langle f-g, f-g\rangle d v<\varepsilon
$$

and $\bar{\partial} f=0$.

Proof) By the Hahn-Banach's theorem it suffices to show that if $u \in L^{n, q}\left(X_{0}, B\right)$ and

$$
\int_{X_{0}}\langle f, u\rangle d v=0
$$

for any $f \in L_{l o c}^{n, q}(X, B)$ with $\bar{\partial} f=0$, then we have

$$
\int_{X_{0}}\langle g, u\rangle d v=0
$$

if $g \in L^{n, q}\left(X_{0}, B\right)$ and $\vec{\partial} g=0$.

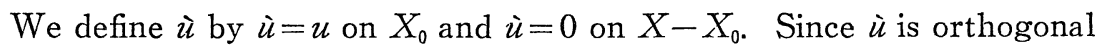
to $N_{\overline{0}}^{n, q}$ for any $\nu$, we have $\grave{\iota} \in R_{v_{\nu}, \bar{q}}^{n, \quad} \quad R_{v_{\nu}, q}^{n, q} \overline{R_{v}^{n}, q}$ is equivalent to $R_{\bar{\partial}}^{n, q+1}$ $=\overline{R_{\bar{\partial}}^{n, \bar{q}+1}}$ (See [2], Theorem 1.1.1). $\quad R_{\bar{\partial}}^{n, q+1}=\overline{R_{\bar{\partial}}^{n, q+1}}$ is proved for $\nu \geq 0$ similarly as Theorem 2.6. Hence, by Proposition 4.2 there exists $\nu_{0}$ such that

$$
\begin{aligned}
& \grave{u}=\vartheta_{\nu} v_{\nu}, \quad \text { for some } v_{\nu} \in L^{n, q+1}(X, B, \nu \Psi), \\
& \text { with }\left\|v_{\nu}\right\|_{\nu}^{2} \leq C_{0}\|\grave{u}\|^{2}, \quad \text { for } \nu \geq \nu_{0} .
\end{aligned}
$$

We set

$$
w_{\nu}=e^{-\nu T} v
$$

then as in the proof of Proposition 4.2, $\left\{w_{\nu}\right\}$ has a subsequence which is weakly convergent in $L^{n, q+1}(X, B)$. Let the weak limit be $w$, then as in the proof of Proposition 4.2, $\vartheta w=\grave{\iota}$ and supp $w \subset \bar{X}_{0}$, hence $\bar{\partial}^{*}\left(\left.w\right|_{X_{0}}\right)=u$. Therefore, if $g \in L^{n, q}\left(X_{0}, B\right)$ and $\bar{\partial} g=0$, we have

$$
\int_{X_{0}}\langle g, u\rangle d v=\int_{X_{0}}\langle\bar{\partial} g, w\rangle d v=0
$$

q.e.d.

Theorem 4.6. The natural map

$$
\rho_{\pi}: H^{n, q}(X, B) \rightarrow H^{n, q}\left(X_{d}, B\right)
$$


is an isomorphism if $q \geq 1$ and $d>0$.

Proof) We consider the following diagram

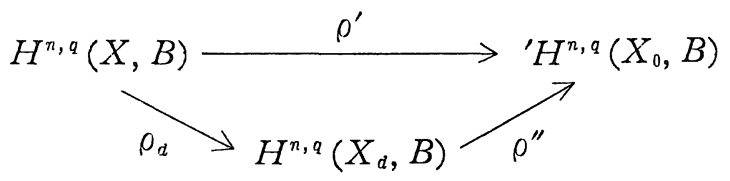

Since $\rho^{\prime}$ is injective $\rho_{d}$ is injective. To show the subjectivity of $\rho_{d}$, we have only to show that $\operatorname{Im} \rho^{\prime}=\operatorname{Im} \rho^{\prime \prime}$, where $\operatorname{Im} \rho^{\prime}$ denotes the image of $\rho^{\prime}$.

By Theorem 4.6 (and by the Dolbeault's theorem), Im $\rho^{\prime}$ is dense in $\operatorname{Im} \rho^{\prime \prime}$. Since $\operatorname{Im} \rho^{\prime \prime}$ is a finite dimensional subspace of the Hilbert space ' $H^{n, q}\left(X_{0}, B\right)$, we have

$$
\operatorname{Im} \rho^{\prime}=\operatorname{Im} \rho^{\prime \prime} .
$$

Thus $\rho_{d}$ is an isomorphism.

q.e.d.

\section{§ 5. Application to Analytic Geometry}

Let $M$ be a complex manifold and $S$ be a nonsingular divisor on $M$ with a proper and smooth holomorphic map $p: S \rightarrow D$ onto a Stein manifold $D$.

\section{Proposition 5. 1 .}

Assumption:

1) $\left.[S]\right|_{p^{-1}(x)}$ is a negative line bundle for any $x \in D$.

2) There is a compact subset $K \Subset D$ such that $\Omega_{S}^{n}$ is negative on $p^{-1}(D-K)$. Here $n=\operatorname{dim} S$ and $\Omega_{S}^{n}$ is the sheaf of holomorphic $n$-forms on $S$.

Conclusion: $S$ is contractible to $D$ in $M$, namely, there is a neighbourhood $V$ of $S$, an analytic space $U$ containing $D$ as a closed analytic subset, and a proper surjective holomorphic map $\varpi: V \rightarrow U$ such that $\left.\varpi\right|_{S}=p$ and $\left.\varpi\right|_{V-S}: V-S \rightarrow U-D$ is biholomorphic.

Proof) By assumption 1), there is a neighbourhood $V^{(2)}$ and a $C^{\infty}$ 
plurisubharmonic function $\Psi^{(2)}$ such that

$$
\Psi^{(2)}(x)=0 \quad \text { if } \quad x \in S,
$$

and $\left.\Psi^{(2)}\right|_{V^{(2)}-S}$ is strictly plurisubharmonic with positive values, (see [1], 4). By 1) and 2) $p^{-1}(D-K)$ is contractible to $D-K$ in a neighbourhood of $p^{-1}(D-K)$, (see [1], Theorem 1$)$. Let the contraction be

$$
\begin{aligned}
& \varpi^{\prime}: V^{*} \longrightarrow U^{*} \\
& p^{-1}(D-L) \longrightarrow D-K \text {. }
\end{aligned}
$$

Since $D$ is a Stein manifold $D$ is properly embedded into some $\mathbb{C}^{N}$. Let $\psi$ be the restriction of $\sum_{i=1}^{N}\left|z_{i}\right|^{2}$ to $D$. Applying Richberg's theorem ([7], Satz 3.3) to $D-K$ and $U^{*}$, we obtain a neighbourhood $U^{* *}$ of $D-K$ and a $C^{\infty}$ strongly pseudoconvex function $\varphi$ on $U^{* *}$ such that $\left.\varphi\right|_{D-K}$ $=\psi$.

Let $c=\sup _{x \in K} \psi(x), d_{1}>d_{2}>c, \varepsilon>0 V^{(1)}=\left\{x ; x \in V^{(2)}, \Psi^{(2)}(x)<\varepsilon\right\}$, and $V$ be the union of connected components of $V^{(1)}-\left\{x ; x \in \varpi^{\prime-1}\left(U^{* *}\right)\right.$, $\left.\psi \circ \bar{w}^{\prime}(x) \geq d_{1}\right\}$ that meet $\left\{x ; x \in S, \varphi \circ p(x)<d_{2}\right\}$, then $V$ is weakly 1 complete for sufficiently small $\varepsilon$.

By 1) and 2), $\left.\Omega_{V}^{n+1} \otimes[S]\right|_{V} ^{-1}$ is positive outside a compact subset. Thus, by Theorem 4.5, $H^{1}\left(V,\left.[S]\right|_{v} ^{-1}\right)$ is finite dimensional. Therefore, for any compact subset $Q$ in $\left\{x ; x \in S, \varphi \circ p(x)<d_{2}\right\}$ we can choose an analytic polyhedron $P$ such that $Q \subset P \subseteq\left\{x ; x \in S, \varphi \circ p(x)<d_{2}\right\}$ and the functions defining $P$ are holomorphic functions on $\left\{x ; x \in S, \varphi \circ p(x)<d_{2}\right\}$ which are restrictions of holomorphic functions on $V$. Since $\partial V_{c}$ is strongly pseudoconvex outside $S$ for almost all $c>0$, this proves that $V$ is holomorphically convex, ([3], Satz 3.4). It is clear that $V-S$ does not contain a compact analytic subset whose dimension is greater than 1 . Consequently, $S$ is contractible to $D$ in $M$.

q.e.d.

Theorem 5.2. Let $X$ be a weakly 1-complete manifold. If there is a holomorphic line bundle $\pi: B \rightarrow X$ which is positive outside a compact subset of $X$, then there is a meromorphic map $\iota: X_{c} \rightarrow \boldsymbol{P}^{N}$, where $c>0$ and $N$ is a natural number depending on $c$, such that there exists a compact analytic set $A \subset X$ such that $\left.\iota\right|_{X_{0}-A}: X_{c}-A \rightarrow \mathbb{P}^{N}$ is a holomorphic imbedding of $X_{c}-A$ as a locally closed analytic 
subset of $\boldsymbol{P}^{N}$.

Proof) Similar as Kodaira's embedding theorem ([4]).

\section{Appendix}

We let the notations be as before.

Lemma If $g \in L^{p, q+1}(X, B), \vartheta g \in L^{p, q}(X, B)$ and supp $g \subset \bar{X}_{0}$ then $\left.g\right|_{X_{0}} \in D_{\bar{\partial}^{*}}$ where $\bar{\partial}^{*}$ is the adjoint of $\bar{\partial}^{*}: L^{p, q}\left(X_{0}, B\right) \rightarrow L^{p, q+1}\left(X_{0}, B\right)$

Proof) Since $\bar{X}_{0}$ is compact and $\partial X_{0}$ is smooth, there is a sequence $\left\{g_{\nu}\right\}$ such that $g_{\nu} \in C_{0}^{p, q+1}(X, B), \quad \operatorname{supp} g_{\nu} \subset X_{0}, \quad \lim _{\nu \rightarrow \infty}\left\|g_{\nu}-g\right\|=0$, and $\lim \left\|\vartheta g_{\nu}-\vartheta g\right\|=0$ (cf. [2], Proposition 1.2.3).

Thus, if $u \in L^{p, q}\left(X_{0}, B\right)$ and $\bar{\partial} u \in L^{p, q+1}\left(X_{0}, B\right)$,

$$
\begin{aligned}
& \int_{X_{0}}\langle u, \vartheta g\rangle d v=\lim _{\nu \rightarrow \infty} \int_{X_{0}}\left\langle u, \vartheta g_{\nu}\right\rangle d v \\
& \quad=\lim _{\nu \rightarrow \infty} \int_{X_{0}}\left\langle\bar{\partial} u, g_{\nu}\right\rangle d v=\int_{X_{0}}\langle\bar{\partial} u, g\rangle d v .
\end{aligned}
$$

Hence $\left.g\right|_{X_{0}} \in D_{\bar{\partial}^{*}}$ and $\bar{\partial} *\left(\left.g\right|_{X_{0}}\right)=\left.\vartheta g\right|_{X_{0}}$.

q.e.d.

\section{References}

[1] Cornalba, M., Two theorems on modifications of analytic spaces, Inventiones Math., 20, (1973) 227-247.

[2] Hörmander, L., $L^{2}$ estimates and existence theorems for the $\bar{\partial}$ operator, Acta Math., 113, (1965) 89-152.

[3] Knorr, K. and Schneider, M., Relativexzeptionelle analytische Mengen, Math. Ann., 193, (1971) 238-254.

[4] Kodaira, K., On kähler varieties of restricted type, Ann. Math., 60, (1954) 28-48.

[5] Nakano, S., On the inverse of monoidal transformation, Publ. RIMS, Kyoto Univ., 6, (1970/71) 483-592.

[6] - Vanishing theorems for weakly 1-complete manifolds, II. Publ. RIMS, Kyoto Univ., 10, (1974) 101-110.

[7] Richberg, R., Stetige streng pseudokonvexe Funktionen, Math. Annalen, 175, (1968) 257-286.

[8] Vesentini, E., Lectures on Levi convexity of complex manifolds and cohomology vanishing theorems, Tata Institute of Fundamental Research, Bombay, 1967.

[9] Weil, A., Introduciton à l'étude des variétés kähleriennes, Act. Sci. Ind. 1267, Hermann, 1958.

Added in Proof: The author proved in a forthcoming paper ' $O n H^{p} \cdot \mathrm{q}(\mathrm{X}, \mathrm{B})$ of weakly 1-complete maniforlds' the finte dimensionality of $\mathrm{H}^{\mathrm{p}, \mathrm{q}}(\mathrm{X}, \mathrm{B})$ and bijectivity of $\mathrm{H}^{\mathrm{p}, \mathrm{q}}(\mathrm{X}, \mathrm{B})$ $\rightarrow \mathrm{H}^{p, q}\left(X_{d}, B\right)$ in the case of $p+q>\operatorname{dim} X$. 